\title{
ARTICLE Sulfation predominates the pharmacokinetics, metabolism, and excretion of forsythin in humans: major enzymes and transporters identified
}

\author{
Lu-lu Pan ${ }^{1,2}$, Yong Yang ${ }^{3}$, Min Hui ${ }^{4}$, Shuo Wang ${ }^{4}$, Cui-yun $\mathrm{Li}^{5}$, Hong Zhang ${ }^{5}$, Yan-hua Ding ${ }^{5}$, Li Fu ${ }^{4}$, Xing-xing Diao ${ }^{1,2}$ and \\ Da-fang Zhong ${ }^{1,2}$
}

Forsythin extracted from Forsythiae Fructus is widely used to treat fever caused by the common cold or influenza in China, Japan and Korea. The present study aimed to analyze the pharmacokinetics, metabolism and excretion routes of forsythin in humans and determine the major enzymes and transporters involved in these processes. After a single oral administration, forsythin underwent extensive metabolism via hydrolysis and further sulfation. In total, 3 of the 13 metabolites were confirmed by comparison to reference substances, i.e., aglycone $M 1, M 1$ sulfate (M2), and $M 1$ glucuronide (M7). Hydrolysis was the initial and main metabolic pathway of the parent compound, followed by extensive sulfation to form $M 2$ and a reduced level of glucuronidation to form $M 7$. In addition, the plasma exposure of M2 and M7 were 86- and 4.2-fold higher than that of forsythin. Within $48 \mathrm{~h}, \sim 75.1 \%$ of the administered dose was found in urine, with $\mathrm{M} 2$ accounting for $71.6 \%$. Further phenotyping experiments revealed that sulfotransferase $1 \mathrm{~A} 1$ and UDP-glucuronosyltransferase $1 \mathrm{~A} 8$ were the most active hepatic enzymes involved in the formation of $M 2$ and M7, respectively. The in vitro kinetic study provided direct evidence that M1 showed a preference for sulfation. Sulfated conjugate M2 was identified as a specific substrate of organic anion transporter 3, which could facilitate the renal excretion of M2. Altogether, our study demonstrated that sulfation dominated the metabolism and pharmacokinetics of forsythin, while the sulfate conjugate was excreted mainly in the urine.

Keywords: forsythin; pharmacokinetics; drug metabolism; drug excretion; sulfation; glucuronidation; organic anion transporter 3

Acta Pharmacologica Sinica (2021) 42:311-322; https://doi.org/10.1038/s41401-020-0481-8

\section{INTRODUCTION}

Forsythin is a compound extracted from Forsythiae Fructus (FF, Lianqiao in Chinese) [1]. FF is widely used as an antipyretic agent in China, Japan and Korea [2]. According to the Pharmacopoeia of China 2015 Edition, there are at least 114 Chinese herbal preparations with FF as an active ingredient, including Shuanghuanglian oral solution, Niuhuang Shangqing tablet, and Yinqiao Jiedu tablet etc [3]. Forsythin belongs to a class of natural glycosidic lignan compounds [4], and can be used to treat fevers caused by the common cold or influenza. Phase II clinical trials of forsythin are currently under way in China (http://www. chinadrugtrials.org.cn/, registration number: CTR20192482), and the proposed dosing regimen is 100 or $200 \mathrm{mg}$ TID.

Sulfation and glucuronidation are considered to be common and important phase II metabolic reactions mediated by sulfotransferases (SULTs) and UDP glucuronic acid transferases (UGTs). The majority of phenolic compounds are sensitive to extensive sulfation and glucuronidation. Typically, sulfation displays high affinity and low capacity for xenobiotic conjugation, while glucuronidation acts in the opposite way. For instance, with increasing dose, the percent acetaminophen that is conjugated with sulfate decreases, while that conjugated with glucuronic acid increases. In addition, UGT-mediated glucuronidation is generally considered to be the main pathway of polyphenol metabolism [5], whereas SULT-mediated sulfation is often regarded as the secondary pathway [6].

Therefore, this study aimed to (1) determine the metabolism of forsythin in humans; (2) analyze the pharmacokinetic profiles and excretion routes of forsythin and its metabolites in humans; (3) evaluate the roles of metabolizing enzymes in forsythin biotransformation; and (4) assess the roles of transporters in forsythin excretion.

\section{MATERIALS AND METHODS}

Chemicals

The forsythin reference substance (purity 94.9\%) was supplied by the National Institute for Food and Drug Control (Beijing, China). The hydrolyzed metabolite aglycone (M1, purity $99.5 \%)$, M1 sulfate (M2, purity $99.5 \%)$, and M1 glucuronide (M7, purity 96.6\%) were synthesized by Dalian Fusheng Pharmaceutical Co., Ltd (Dalian, China). The structures of these four reference substances were

\footnotetext{
${ }^{1}$ State Key Laboratory of Drug Research, Shanghai Institute of Materia Medica, Chinese Academy of Sciences, Shanghai 201203 , China; ${ }^{2}$ University of Chinese Academy of Sciences, Beijing 100049, China; ${ }^{3}$ Suzhou Haike Medical Technology Co., Ltd., Suzhou 215123, China; ${ }^{4}$ Dalian Fusheng Pharmaceutical Co., Ltd., Dalian 116000 , China and ${ }^{5}$ The First Hospital of Jilin University, Changchun 130021, China

Correspondence: Yan-hua Ding (dingyanhua2003@126.com) or Li Fu (2729677386@qq.com) or Xing-xing Diao (xxdiao@simm.ac.cn) or Da-fang Zhong (dfzhong@simm.ac.cn)
}

Received: 31 March 2020 Accepted: 13 July 2020

Published online: 28 August 2020 
312

confirmed based on nuclear magnetic resonance (NMR) and mass spectrometry (MS) data (Supplementary file).

The internal standard teniposide (TNP) was supplied by Tokyo Chemical Industry Co., Ltd. (Tokyo, Japan). The other internal standard, C52241 (used for the quantitative analyses of urine and fecal samples), was kindly provided by Dr. Jing-shan Shen's laboratory, Shanghai Institute of Materia Medica, Chinese Academy of Sciences. Probenecid, aclacinomycin, Hanks' balanced salt solution (HBSS), uridine-5'-diphospho-glucuronic acid (UGDPA), and 3'-phosphoadenosine-5'-phosphosulfate (PAPS) were supplied by Sigma-Aldrich Co. (St. Louis, MO, USA). Metformin, paraaminohippurate (PAH) and estrone-3-sulfate (E3S) were supplied by the National Institute for Food and Drug Control, MCE (Monmouth Junction, NJ, USA) and TCL Pharma (Toronto, Canada). Penicillin, streptomycin and $0.05 \%$ trypsin-EDTA were obtained from Gibco (Carlsbad, CA, USA). The bicinchoninic acid (BCA) protein assay kit was purchased from Beyotime (Shanghai, China). Fetal bovine serum (FBS), poly- $D$-lysine-coated plates, Dulbecco's modified Eagle's medium (DMEM) and recombinant human UGT1A1, 1A3, 1A4, 1A6, 1A7, 1A8, 1A9, 1A10, 2B4, 2B7, 2B15, and $2 B 17$ isoenzymes were purchased from Corning Gentest (Woburn, MA, USA). Recombinant human SULT1A1*1, 1A1*2, 1A2, 1A3, 1B1, 1E1, and 2A1 were supplied by Cypex (Dundee, Scotland, UK). Purified and deionized water was prepared using a Milli-Q gradient water purification system (Millipore, Molsheim, France). All other solvents and reagents were of either highperformance liquid chromatography (HPLC) or analytic grade.

Research protocol and sample collection

This was a single-center, randomized, double-blind, single ascending dose phase I study to evaluate the pharmacokinetics of metabolism of forsythin in healthy subjects. The clinical study was conducted in the Phase I Clinical Trial Unit, First Hospital of Jilin University. Ethical approval for this trial protocol was obtained from the Ethics Committee of the First Hospital of Jilin University (Changchun, China). The design of clinical research monitoring was in compliance with the Declaration of Helsinki and in accordance with the guidelines of Good Clinical Practice. Written informed consent was obtained from all the study participants before the initiation of the study procedures. Sixty-two healthy volunteers aged $18-45$ were recruited. A total of six dose groups were set up, which were $50,100,200,400,600$, and $800 \mathrm{mg}$ of forsythin. Two additional subjects as a pilot group were enrolled before the trial in the $50 \mathrm{mg}$ dose group. The blood sample collection time was adjusted based on the pharmacokinetic results of these two subjects. There were 10 subjects in each group, of which eight received the test drug and the other two received placebo; the ratio of males to females was close to 1. A summary of the demographics of the study subjects is shown in Supplementary Table S1.

After overnight fasting, eight healthy volunteers from each group were orally administered forsythin capsules in the morning (50 mg/capsule). Different groups received different doses with $240-480 \mathrm{~mL}$ of water (e.g., the $800 \mathrm{mg}$ group received 16 capsules). Blood sample collection was performed at 0 (predose), $0.25,0.5,0.75,1,1.5,2,2.5,3,4,6,8,10,12,14,16,24$, and $36 \mathrm{~h}$ postdose (hpd). The design of the study is shown in Supplementary Fig. S1. After $72 \mathrm{~h}$ of hospitalization, if forsythin is safe for the subjects, then we can increase the dose. For the excretion study of forsythin and its metabolites, urine and feces were collected in the $100 \mathrm{mg}$ dose group. Urine sample collection was performed at 0 (predose), 0-4, 4-8, 8-12, 12-24, 24-48, and 48-72 hpd. Fecal sample collection was performed at 0 (predose) and 0-72 hpd. All samples were preserved at $-70^{\circ} \mathrm{C}$ until further analysis.

Metabolite profiling and identification

Plasma and urine sample preparation. All plasma samples (predose and $4 \mathrm{hpd}$ ) from three subjects of the same sex were pooled at equal volumes. Urine samples (predose and 0-48 hpd) were pooled by using the volumes in proportion to the overall volume across the time intervals. Approximately $600 \mu \mathrm{L}$ of acetonitrile was added to $200 \mu \mathrm{L}$ of the pooled plasma and urine samples, followed by vortexing for $1 \mathrm{~min}$. Then, the mixture was centrifuged at $14,000 \mathrm{rpm}$ for $5 \mathrm{~min}$. Subsequently, the supernatant was transferred into a $10 \mathrm{~mL}$ tube and evaporated to dryness at $40^{\circ} \mathrm{C}$ under a stream of nitrogen. The residue was reconstituted in $120 \mu \mathrm{L}$ of acetonitrile/water $(5: 95, \mathrm{v} / \mathrm{v})$, and the resulting solution $(7 \mu \mathrm{L})$ was subjected to ultra-performance liquid chromatography/quadrupole time-of-flight mass spectrometry (UPLC/Q-TOF MS) analysis.

Fecal sample preparation. The fecal samples were thawed and then weighed. Approximately $10 \mathrm{~mL}$ of methanol/water $(1: 1, \mathrm{v} / \mathrm{v})$ was added to $2 \mathrm{~g}$ of a fecal sample, followed by blending for $10 \mathrm{~min}$ and sonication for $20 \mathrm{~min}$. The fecal samples (predose and 0-24 hpd) from three subjects of the same sex were pooled. Then, $200 \mu \mathrm{L}$ of the fecal homogenate was mixed with $600 \mu \mathrm{L}$ of acetonitrile for $1 \mathrm{~min}$. After centrifugation at 14,000 rpm for $5 \mathrm{~min}$, the supernatant was evaporated to dryness at $40^{\circ} \mathrm{C}$ under nitrogen. Finally, the residue was reconstituted in $120 \mu \mathrm{L}$ of acetonitrile/water $(5: 95, v / v)$, and the resulting solution was subjected to UPLC/Q-TOF MS analysis.

UPLC/Q-TOF MS analysis. Chromatographic separation was performed on an Acquity UPLC HSS T3 column $(1.8 \mu \mathrm{m} ; 100 \mathrm{~mm} \times$ $2.1 \mathrm{~mm}$ ) using an Acquity UPLC system (Waters, Milford, MA, USA). The mobile phase consisted of $5 \mathrm{mM}$ ammonium acetate $[0.01 \%$ ammonia] (A) and acetonitrile (B). The gradient elution was maintained at 5\% B for 2 min, increased linearly to $60 \%$ B over $10 \mathrm{~min}$ and held for $2 \mathrm{~min}$, further increased to $95 \% \mathrm{~B}$ over the next $1 \mathrm{~min}$, and ultimately reverted back to $5 \% \mathrm{~B}$ for $2 \mathrm{~min}$. The column temperature and flow rate were fixed at $40{ }^{\circ} \mathrm{C}$ and $0.4 \mathrm{~mL} /$ min, respectively. Fractions were collected during elution and detected by UV at $280 \mathrm{~nm}$.

MS analysis was conducted with a Synapt G2-Si Q-TOF highresolution mass spectrometer (Waters, Milford, MA, USA) in negative electrospray ionization (ESI) mode. The desolvation gas flow was optimized to $800 \mathrm{~L} / \mathrm{h}$ at $450^{\circ} \mathrm{C}$, with the capillary voltage set at $3.0 \mathrm{kV}$ and source temperature fixed at $120^{\circ} \mathrm{C}$. At the time of acquisition, data were centroided within the range of $50-1200 \mathrm{Da}$ using $50 \mathrm{ng} / \mathrm{mL}$ leucine enkephalin infused at $10 \mu \mathrm{L} / \mathrm{min}$ in order to produce a reference ion at $\mathrm{m} / \mathrm{z} 554.2615$. The $\mathrm{MS}^{\mathrm{E}}$ scan function was used to simultaneously analyze both precursor and fragment ions in a single run by rapidly alternating between two independent collision energy (CE) parameters. At low CE background, the transfer and trap CEs were 2 and $5 \mathrm{~V}$, respectively. At high CE background, the transfer CE was $15 \mathrm{~V}$, while the trap $C E$ ramped from 10 to $20 \mathrm{~V}$. Data collection and analysis were conducted by MassLynx software version 4.1 and UNIFI 1.8.2 software (Waters, Milford, MA, USA), respectively.

\section{Pharmacokinetics of forsythin and its metabolites in human} plasma

Analytical method. The plasma samples were kept frozen at $-70^{\circ} \mathrm{C}$ and then analyzed during storage. The plasma concentrations of forsythin and its main metabolites $M 1, M 2$, and $M 7$ were assessed using an LC-MS/MS method validated based on the Chinese Pharmacopoeia guidelines [7]. After removing proteins through precipitation with methanol, the analytes and internal standard teniposide were eluted using a Gemini $C_{18}$ column $(110 \AA, 2.0 \mathrm{~mm} \times 50 \mathrm{~mm}, 5 \mu \mathrm{m}$; Phenomenex). The mobile phase consisted of methanol-acetonitrile $(2: 1, v / v)$ and $0.01 \%$ ammonia in water with gradient elution. The total run time was $5.5 \mathrm{~min}$. MS detection was performed in ESI (-) multiple reaction monitoring mode. The calibration curves were linear over the plasma concentration ranges of 1.00-1000, 0.500-500, 5.00-5000, and 
2.50-2500 ng/mL for forsythin, $M 1, M 2$, and $M 7$, respectively. The lower limits of quantification for forsythin, M1, M2, and M7 were $1.00,0.500,5.00$, and $2.50 \mathrm{ng} / \mathrm{mL}$, respectively.

Calculation of pharmacokinetic parameters. WinNonlin (V7.0, Pharsight, Mountain View, CA, USA) with noncompartmental analysis was employed to calculate the pharmacokinetic parameters. The maximum plasma concentration $\left(C_{\max }\right)$ and the time to achieve $C_{\max }\left(T_{\max }\right)$ were obtained directly from the pharmacokinetic data. The area under the plasma concentration-time curve (AUC) was calculated according to the linear trapezoidal with linear interpolation method to the last measurable concentration $\left(A \cup C_{0-t}\right)$. The AUC from zero to infinity $\left(A \cup C_{0-\infty}\right)$ was calculated by $A \cup C_{0-\infty}=A \cup C_{0-t}+C_{\text {last }} / \lambda_{z}$ ( $C_{\text {last }}$, the last measurable drug concentration, and $\lambda_{z}$, the terminal elimination rate estimated by log-linear regression of plasma concentrations observed during the terminal phase of elimination). The apparent elimination half-life $\left(t_{1 / 2}\right)$ was calculated as $t_{1 / 2}=\ln 2 / \lambda_{z}$. Other pharmacokinetic parameters included the apparent volume of distribution $\left(\mathrm{V}_{\mathrm{Z}} / \mathrm{F}\right)$ and apparent clearance $(\mathrm{CL} / \mathrm{F})$, which was calculated by $\mathrm{CL} / \mathrm{F}=$ dose $/ \mathrm{AUC}_{0-\infty}$. $T_{\max }$ was expressed as the median with interquartile range; all other parameters are expressed as the mean \pm standard deviation (SD).

Excretion of forsythin and its metabolites in human urine and feces

The urine and fecal concentrations of forsythin, M1, M2, and M7 were assessed using the validated LC-MS/MS method. C52241 was chosen as the internal standard at a concentration of $500 \mathrm{ng} / \mathrm{mL}$. Briefly, the analytes and internal standard were separated using a BEH $\mathrm{C}_{18}$ column $(1.7 \mu \mathrm{m}, 50 \times 2.1 \mathrm{~mm}$, Waters $)$ after methanolbased protein precipitation. The mobile phase was composed of methanol and $2 \mathrm{mM}$ ammonium acetate with a gradient elution program. A total run time of $2.6 \mathrm{~min}$ was used for chromatographic separation at a $0.6 \mathrm{~mL} / \mathrm{min}$ flow rate. The standard curves ranged from 10.0 to $5000,10.0$ to $5000,20.0$ to 10,000 , and 10.0 to $5000 \mathrm{ng} / \mathrm{mL}$ for forsythin, M1, M2, and M7, respectively, in human urine, while the standard curves ranged from 25.0 to $12500,50.0$ to $25,000,150$ to 75,000 , and 25.0 to $12,500 \mathrm{ng} / \mathrm{g}$ for forsythin, M1, $M 2$, and $M 7$, respectively, in human feces. Finally, the cumulative urinary and fecal excretion rates of the drug were calculated.

Human SULT isoenzyme phenotyping

The total volume for the incubation system was $200 \mu \mathrm{L}$ and consisted of $10 \mathrm{mM}$ PBS ( $\mathrm{pH} 7.4$ ), $250 \mu \mathrm{M}$ PAPS, $50 \mu \mathrm{M} \mathrm{M} 1$, and $0.25 \mathrm{mg} / \mathrm{mL}$ human recombinant SULT isoform (SULT1A $1{ }^{*} 1$, $1 A 1 * 2,1 A 2,1 A 3,1 B 1,1 E 1$, or $2 A 1)$. Before adding the recombinant SULT isoform, preincubation was conducted at $37^{\circ} \mathrm{C}$ for $3 \mathrm{~min}$. After $1 \mathrm{~h}$ of reaction, an equal amount of ice-cold acetonitrile was added to terminate the reaction. All assays were carried out in triplicate. The final concentration of organic solvent in all reactions was $<0.1 \%$. All phenotyping samples were kept at $-70^{\circ} \mathrm{C}$ prior to analysis.

Inhibition of the sulfation reaction of M1 in human liver cytosols (HLCs) by the SULT1A1- and SULT1E1-sensitive inhibitor quercetin and SULT inhibitor 2,6-dichloro-4-nitrophenol (DCNP) was performed. The other conditions were similar to those described above. Sulfation of M1 in inhibited samples was compared with that of the control group to determine the remaining enzyme activity.

\section{Human UGT isoenzyme phenotyping}

The total volume for the incubation system was $100 \mu \mathrm{L}$, containing $50 \mathrm{mM}$ Tris- $\mathrm{HCl}$ buffer (pH 7.5), $50 \mu \mathrm{M} \mathrm{M1}, 25 \mu \mathrm{g} / \mathrm{mL}$ aclacinomycin, $2 \mathrm{mM}$ UDPGA, and $0.5 \mathrm{mg} / \mathrm{mL}$ human recombinant UGT isoform (UGT1A1, 1A3, 1A4, 1A6, 1A7, 1A8, 1A9, 1A10, 2B4, 2B7, $2 \mathrm{~B} 15$, or $2 \mathrm{~B} 17)$. Before adding the recombinant UGT isoform, preincubation was conducted at $37^{\circ} \mathrm{C}$ for $3 \mathrm{~min}$. After $1 \mathrm{~h}$ of reaction, an equal amount of ice-cold acetonitrile was added to terminate the reaction. All assays were carried out in duplicate. The final concentration of organic solvent in all reactions was $<0.1 \%$. All phenotyping samples were kept at $-70^{\circ} \mathrm{C}$ prior to analysis.

The effect of the general UGT inhibitor flurbiprofen on the formation of M7 was also evaluated using human liver microsomes (HLMs). The other conditions were similar to those described above. Glucuronidation of $M 1$ in inhibited samples was also compared with that of the control group.

Transporter phenotype of sulfation conjugate $\mathrm{M} 2$

Cell culture. Human embryonic kidney 293 (HEK293) cells transfected with human organic anion transporter 1 (OAT1), OAT3 and organic cation transporter 2 (OCT2) as well as emptyvector (mock)-transfected cells were constructed by HD Biosciences Co., Ltd. (Shanghai, China). The functional roles of these three transporters were assessed using the corresponding substrate or inhibitor. The cells were cultured in DMEM containing $10 \% \mathrm{FBS}, 100 \mu \mathrm{g} / \mathrm{mL}$ hygromycin $\mathrm{B}, 100$ units $/ \mathrm{mL}$ penicillin, and $100 \mu \mathrm{g} / \mathrm{mL}$ streptomycin at $37^{\circ} \mathrm{C}, 5 \% \mathrm{CO}_{2}$, and $95 \%$ humidity. Cells were dispensed at a density of $2 \times 10^{5}$ cells/well and grown in a 24-well plate coated with BD Biocoat poly- $D$-lysine. Upon reaching confluency ( 2 days after seeding), cell uptake experiments were carried out.

In vitro uptake experiments. Prior to the in vitro uptake experiments, the cells were washed three times with prewarmed HBSS $\left(\mathrm{pH} 7.4,37^{\circ} \mathrm{C}\right)$ and then incubated with $300 \mu \mathrm{L}$ of HBSS at $37^{\circ} \mathrm{C}$ for $10 \mathrm{~min}$. After removing the pre-equilibrated HBSS solution, the uptake reaction was initiated by adding $300 \mu \mathrm{L}$ of $50 \mu \mathrm{M}$ M2-containing HBSS solution in the presence or absence of inhibitors. The uptake reaction was terminated by removing the solution and rinsing three times with ice-cold HBSS. Cell lysis was performed with $200 \mu \mathrm{L}$ of deionized water after 3 freeze-thaw cycles. To minimize the potential impacts of nonspecific binding and passive uptake, mock-transfected HEK293 cells were included as controls.

Following cell lysis, the solubilized protein samples $(50 \mu \mathrm{L})$, water/methanol (1:1, v/v; $50 \mu \mathrm{L}), \mathrm{C} 52241$ internal standard (500 ng/ $\mathrm{mL} ; 100 \mu \mathrm{L})$, and methanol $(200 \mu \mathrm{L})$ were mixed and vortexed. After centrifugation at $14,000 \mathrm{rpm}$ for $5 \mathrm{~min}$, a $100 \mu \mathrm{L}$ aliquot of the supernatant was diluted with $200 \mu \mathrm{L}$ of water, and $15 \mu \mathrm{L}$ of the resulting solution was subjected to LC-MS/MS analysis. The protein content of the cell lysate was evaluated with a BCA protein assay kit.

The uptake rates of HEK293-OAT1, HEK293-OAT3, HEK293-OCT2, and mock cells were calculated. We considered that active transport occurs when the substrate ratio of the transporter is $>2$ [8]. The formula used for calculation is as follows:

$V_{\text {uptake }}=\left(C_{\text {cell lysate }} \times 1000\right) /\left(t_{\text {uptke }} \times C_{\text {protein }}\right)$

Among these, the unit of the uptake rate $\left(V_{\text {uptake }}\right)$ is pmol. $\cdot \mathrm{min}^{-1} \cdot \mathrm{mg}^{-1}$ protein; $C_{\text {cell lysate }}$ is the level of drug in the cell lysate with the unit of $\mathrm{nM}$; the unit of uptake time $\left(t_{\text {uptke }}\right)$ is minute; and the unit of protein concentration $\left(C_{\text {protein }}\right)$ is $\mu \mathrm{g} / \mathrm{mL}$.

Kinetic analysis of sulfation in SULT1A $1 * 1$ and glucuronidation in UGT1A8

The pre-experiment optimized the reaction times and enzyme concentrations so that metabolite formation was within the linear range and the consumption of substrate M1 did not exceed $20 \%$. The incubation systems were the same as mentioned above (see Human SULT isoenzyme phenotyping and Human UGT isoenzyme phenotyping parts). Nonlinear regression analysis was used to calculate the kinetic parameters $\left(K_{\mathrm{m}}\right.$, the Michaelis constant and $V_{\text {max }}$ the maximum velocity) according to the Michaelis-Menten 

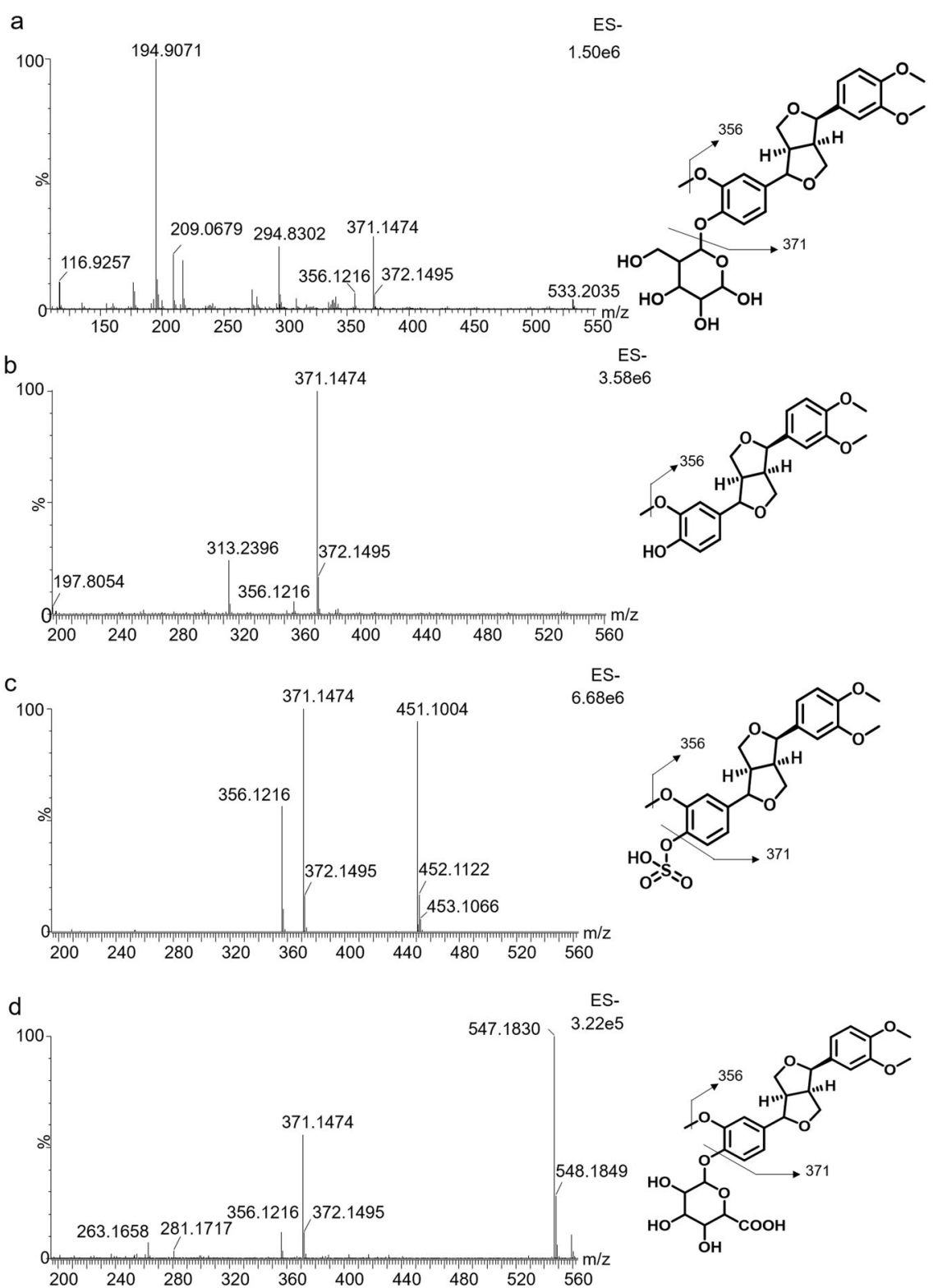

Fig. $1 \mathrm{MS}^{2}$ spectrum of four substances and their proposed fragmentation pathways. a Forsythin; b M1; c M2 and d M7.

equation in GraphPad Prism software 5.0 (GraphPad Software Inc., San Diego, CA, USA).

\section{RESULTS}

UPLC/Q-TOF MS analysis of forsythin

First, the MS/MS fragmentation patterns and chromatographic behaviors of forsythin were studied. The retention time of forsythin was $7.54 \mathrm{~min}$ under the specific chromatographic conditions. In the negative scan mode, forsythin generated a deprotonated molecular ion $[\mathrm{M}-\mathrm{H}]^{-}$at $\mathrm{m} / \mathrm{z}$ 533.2035. The product ion spectrum of forsythin at high collision energy is demonstrated in Fig. 1a. The fragment ions were detected at $\mathrm{m} / \mathrm{z} 371.1474$ $\left(-\mathrm{C}_{6} \mathrm{H}_{10} \mathrm{O}_{5}\right)$ and $356.1216\left(-\mathrm{C}_{6} \mathrm{H}_{10} \mathrm{O}_{5},-\mathrm{CH}_{3}\right)$. Several tentative fragmentation patterns were speculated according to the highresolution mass spectral data (Fig. 1a).

Metabolite profiling and characterization of forsythin After MS data processing with MassLynx V4.1 and UNIFI V1.8.2, the plasma, urine and fecal metabolic profiles of forsythin and its metabolites from healthy volunteers were obtained (Fig. 2). A total of 4,12 , and 3 metabolites were detected from the plasma, urine and fecal samples, respectively. The identities of the three proposed metabolites were verified with reference standards (details are summarized in Table 1). The postulated metabolic pathways of forsythin are illustrated in Fig. 3. Characterization of the metabolites are as follows.

Metabolite M1. M1 eluted at 9.66 min and displayed an $[\mathrm{M}-\mathrm{H}]^{-}$ ion at $m / z$ 371.1484. Its elemental composition was deduced to be $\mathrm{C}_{21} \mathrm{H}_{24} \mathrm{O}_{6}$. Similar to $\mathrm{M0}, \mathrm{M} 1$ exhibited a fragment ion at $\mathrm{m} / \mathrm{z}$ 356.1216 (Fig. 1b). The chromatographic and mass spectrometric behaviors of $\mathrm{M} 1$ were similar to the reference standard, indicating that $\mathrm{M} 1$ is an aglycone metabolite produced from the parent drug via the hydrolysis process.

Metabolite M2. The elemental composition of M2 was inferred to be $\mathrm{C}_{21} \mathrm{H}_{24} \mathrm{O}_{9} \mathrm{~S}$, suggesting the addition of $\mathrm{SO}_{3}$ to $\mathrm{M} 1$. Metabolite $\mathrm{M} 2$ exhibited an $[\mathrm{M}-\mathrm{H}]^{-}$ion at $\mathrm{m} / \mathrm{z} 451.1072$, with an elution time of $6.83 \mathrm{~min}$. The product ion spectrum of $\mathrm{M} 2$ at high collision 


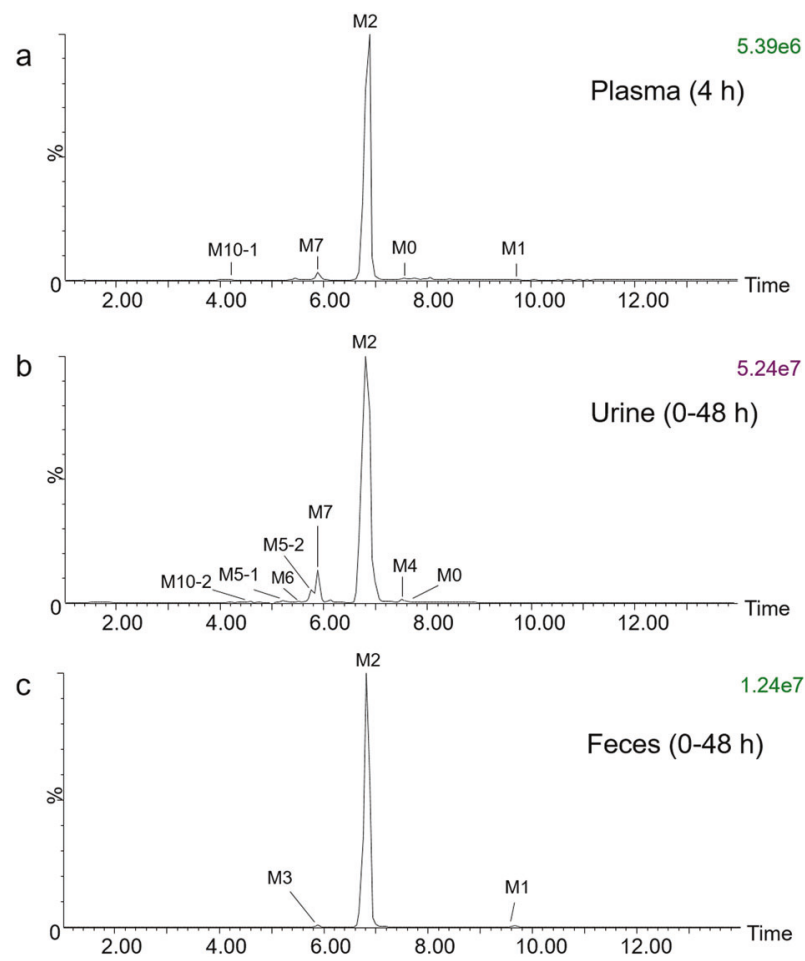

Fig. 2 Metabolic profiles of forsythin in humans $(100 \mathrm{mg}$ dose group). a Pooled plasma at $4 \mathrm{~h}$; b pooled urine at $0-48 \mathrm{~h}$; c pooled feces at $0-48 \mathrm{~h}$.

energy is demonstrated in Fig. 1c. Fragment ions at $m / z 371.1473$ and 356.1216 were detected, which were similar to those of the parent drug. There were high similarities in the chromatographic and mass spectral behaviors of M2 with the reference aglycone sulfate conjugate, confirming that $\mathrm{M} 2$ is a sulfated metabolite of M1.

Metabolite M3. Metabolite M3 displayed an elemental composition of $\mathrm{C}_{21} \mathrm{H}_{24} \mathrm{O}_{10} \mathrm{~S}$, suggesting that an oxygen atom was introduced into $\mathrm{M} 1$. The retention time of the deprotonated ion $[\mathrm{M}-\mathrm{H}]^{-}$at $\mathrm{m} / \mathrm{z} 467.1012$ was $5.89 \mathrm{~min}$. The high-energy MS spectrum of $M 3$ demonstrated fragment ions at $m / z 387.1808$ and 327.1235. The deprotonated ion $[\mathrm{M}-\mathrm{H}]^{-}$of $\mathrm{M} 3$ at $\mathrm{m} / \mathrm{z} 467.1012$ exhibited a mass that was $15.9940 \mathrm{Da}$ greater than that of $\mathrm{M} 2$ at $\mathrm{m} / \mathrm{z}$ 451.1072, signifying the inclusion of an oxygen atom. Therefore, M3 was designated to be a monooxygenated metabolite of $\mathrm{M} 2$ with an uncertain oxidation site.

Metabolite M4. Metabolite M4 (formula: $\mathrm{C}_{26} \mathrm{H}_{32} \mathrm{O}_{11}$ ) exhibited a retention time of $7.28 \mathrm{~min}$ and was generated by the loss of $\mathrm{CH}_{2}$ from M0. The product ion scan spectrum was not collected. Thus, it was presumed that M4 was a demethylated metabolite of M0, and its demethylation site could not be determined.

Metabolite M5. Metabolite M5 displayed an elemental composition of $\mathrm{C}_{26} \mathrm{H}_{30} \mathrm{O}_{12}$. This composition was suggestive of the occurrence of $+\mathrm{O}-2 \mathrm{H}$ from the M4 moiety. M5-1 and M5-2 displayed highly similar ions at $\mathrm{m} / \mathrm{z} 357.1280$ and 357.1360 in the mass spectrum with high collision energy. Hence, M5 could be formed through the demethylation, monooxidation and dehydrogenation of forsythin. However, its metabolic sites remain uncertain.

Metabolite M6. The chromatographic retention time of M6 was $5.35 \mathrm{~min}$, with an $[\mathrm{M}-\mathrm{H}]^{-}$ion at $\mathrm{m} / \mathrm{z} 535.1819$. According to the accurate mass information, the formula of $\mathrm{M} 6$ was inferred to be
$\mathrm{C}_{26} \mathrm{H}_{32} \mathrm{O}_{12}$, suggesting the addition of $\mathrm{O}$ onto the $\mathrm{M} 4$ moiety. It yielded a main fragment ion at $\mathrm{m} / \mathrm{z}$ 359.1530. It could be presumed that M6 was formed through the demethylation and monooxidation of $\mathrm{M} 0$.

Metabolite M7. Metabolite M7 eluted at $5.91 \mathrm{~min}$, which was earlier than its parent drug, suggesting increased polarity. M7 showed an $[\mathrm{M}-\mathrm{H}]^{-}$ion at 547.1819, with an elemental composition of $\mathrm{C}_{27} \mathrm{H}_{32} \mathrm{O}_{12}$. In ESI (-), M7 displayed main fragment ions at $\mathrm{m} / \mathrm{z} 371.1474$ with the neural loss of glucuronic acid (176.0335 Da) and $\mathrm{m} / \mathrm{z} 356.1216$. The product ions of M7 were the same as those of M0. The chromatographic and mass spectrometric behaviors of M7 were similar to the reference standard, indicating that $\mathrm{M7}$ is a glucuronide conjugate of $\mathrm{M} 1$.

Metabolite M8. Metabolite M8 displayed an elemental composition of $\mathrm{C}_{27} \mathrm{H}_{30} \mathrm{O}_{13}$ and eluted at $5.68 \mathrm{~min}$. This composition was suggestive of a $+\mathrm{O}-2 \mathrm{H}$ modification to $\mathrm{M} 7$. The product ion scan spectra of M8 were not collected. Hence, M8 was postulated to be a monooxidated and dehydrogenated metabolite of M7.

Metabolite M9. Metabolite M9 with an elemental composition of $\mathrm{C}_{27} \mathrm{H}_{32} \mathrm{O}_{13}$ was indicative of a monooxygenation product of $\mathrm{M} 7$. The characteristic fragment ion of $\mathrm{M9}$ at $\mathrm{m} / \mathrm{z} 387.1390$ was $15.9916 \mathrm{Da}$ greater than that of $\mathrm{M7}$ at $\mathrm{m} / \mathrm{z}$ 371.1474, further supporting the incorporation of an oxygen atom. Taken together, M9-1 and M9-2 could be generated from the parent drug through the hydroxylation process.

Metabolite M10. The retention times of metabolites M10-1 (found in plasma) and M10-2 (observed in urine) were 4.19 and $4.57 \mathrm{~min}$, respectively. They represented the sulfated conjugates of $\mathrm{M} 5$, as revealed by their elemental composition $\left(\mathrm{C}_{26} \mathrm{H}_{30} \mathrm{O}_{15} \mathrm{~S}\right)$, with increased $\mathrm{SO}_{3}$ compared with M5. M10-1 exerted a main fragment ion at $m / z$ 437.0830, while M10-2 produced a major fragment ion at $\mathrm{m} / \mathrm{z} 533.1650$ under high collision energy. The product ion of M10-1 at $\mathrm{m} / \mathrm{z} 437.0830$ was $79.955 \mathrm{Da}\left(\mathrm{SO}_{3}\right)$ higher than that of M5-1 at $\mathrm{m} / \mathrm{z}$ 371.1474. In addition, the fragment ion of M10-2 at $m / z 533.1650$ was produced by the loss of $79.9586\left(\mathrm{SO}_{3}\right)$ from the precursor ion at $\mathrm{m} / \mathrm{z} 612.1236$. These fragments reveal that M10-1 and M10-2 are the sulfated conjugates of M5.

Pharmacokinetics of forsythin

For the pharmacokinetic analysis, the plasma concentrations of forsythin, M1, M2, and M7 were detected using the abovementioned LC-MS/MS technique. Following a single oral dose administration of $100 \mathrm{mg}$ of forsythin capsules, the absorption and elimination of the drug was rapid. The plasma concentration of the parent drug reached its peak at $1.25 \mathrm{~h}$, with a half-life value of $1.75 \mathrm{~h}$. The plasma concentration-time profiles of forsythin, $\mathrm{M} 1$, $M 2$, and M7 following the administration of 100 and $800 \mathrm{mg}$ forsythin capsules are shown in Figs. 4 and 5, and their respective pharmacokinetic parameters are summarized in Tables 2 and 3, respectively. The plasma concentration-time profiles of the other doses of forsythin, M1, M2, and M7 are presented in Supplementary Figs. S2-S5, and the respective plasma concentration-time profiles are presented in Supplementary Tables S2-S5. At the dose of $100 \mathrm{mg}$, the plasma concentration and exposure of $\mathrm{M} 2$ were the highest. Notably, the peak concentration and $A U C_{0-\infty}$ of $M 2$ were 46- and 86-fold greater than those of the parent drug, respectively. In contrast, the concentration of $\mathrm{M} 1$ was lower, and its peak concentration was $\sim 1 / 10$ that of the parent drug. Nevertheless, the concentration of M7 was higher, and its peak concentration and $\mathrm{AUC}_{0-\infty}$ were 2.3- and 4.2-fold higher than those of the parent drug, respectively. The mean (SD) plasma concentration-time profiles did not show the characteristics of each individual, so three typical concentration-time profiles of healthy volunteers are presented. The clearance values of 
Table 1. Characterization of forsythin metabolites in humans by UPLC/Q-TOF MS.

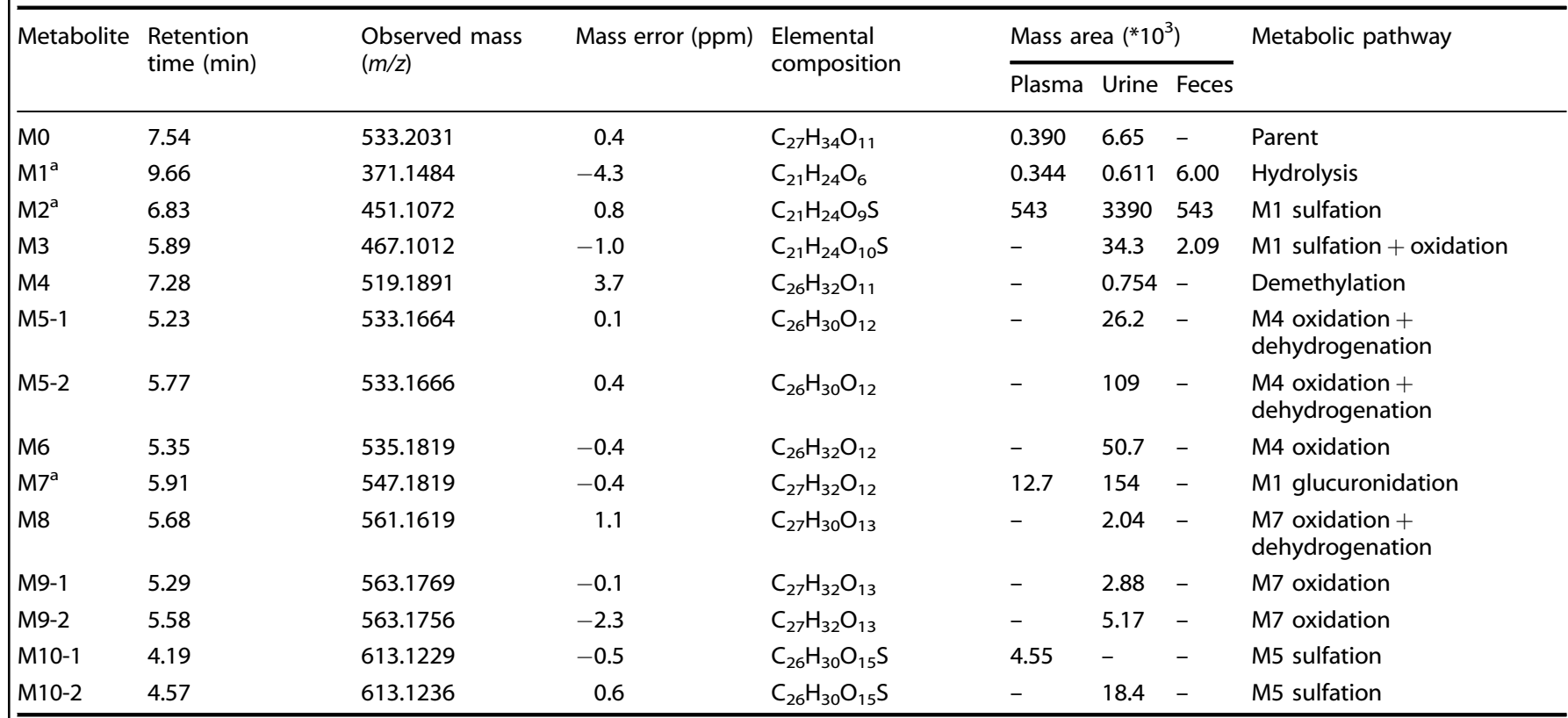

${ }^{\mathrm{a} C o n f i r m e d}$ using reference standards.
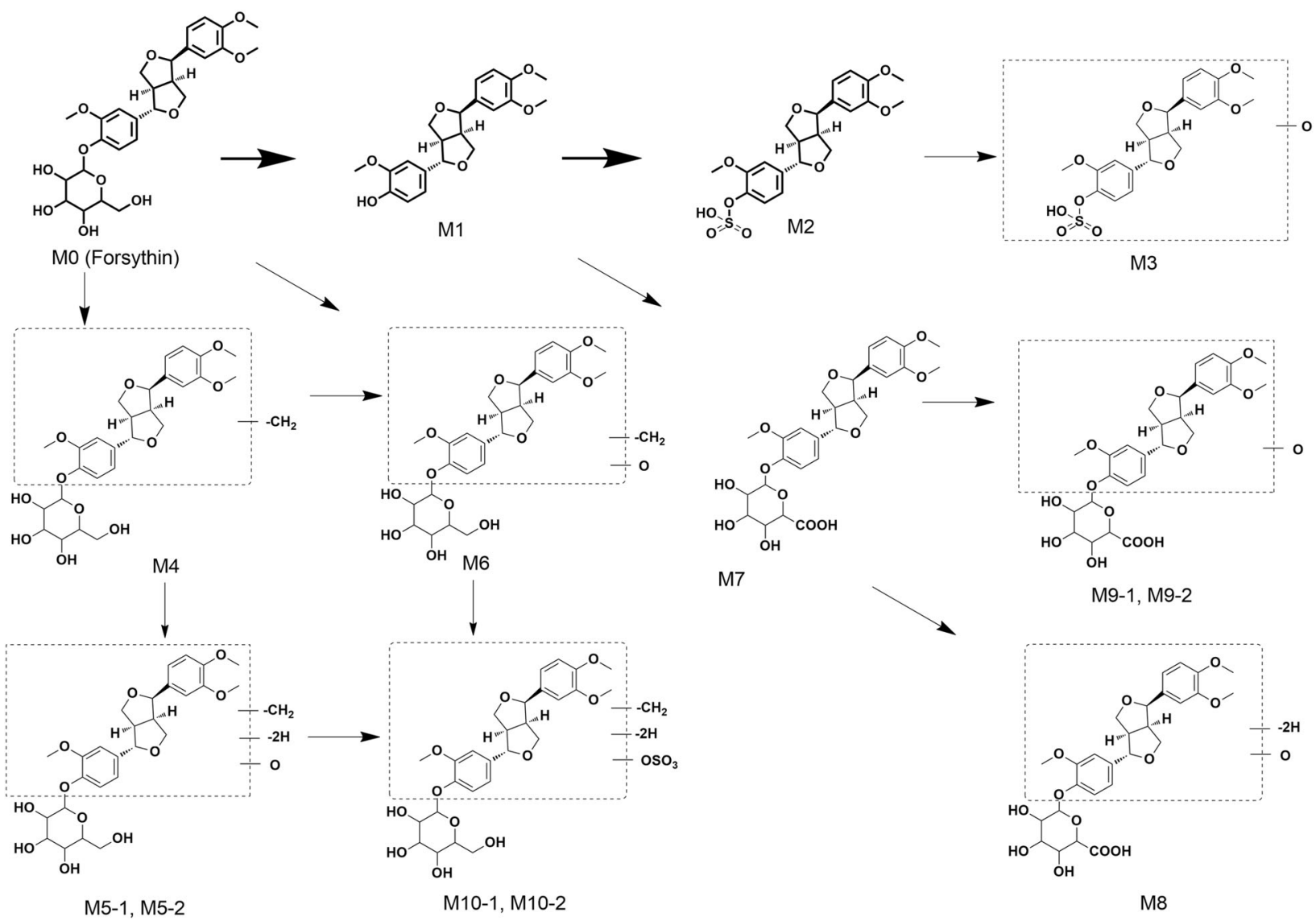

M7

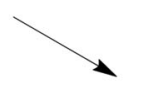

M9-1, M9-2

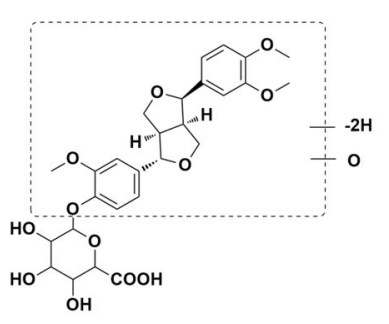

M8

Fig. 3 Proposed metabolic pathways of forsythin in humans.

forsythin were 942 and $3070 \mathrm{~L} / \mathrm{h}$ at doses of 100 and $800 \mathrm{mg}$, respectively. As the dose increased, the $A \cup C_{0-\infty}$ of forsythin increased, but the increase in the $A \cup C_{0-\infty}$ of forsythin was significantly lower than the dose increase rate. The apparent clearance was calculated by $\mathrm{CL} / \mathrm{F}=$ dose/AUC $\mathrm{C}_{-\infty}$. Thus, the clearance values increased nonlinearly with dose. Overall, no serious adverse events were reported throughout the study. No volunteers withdrew because of adverse events. 

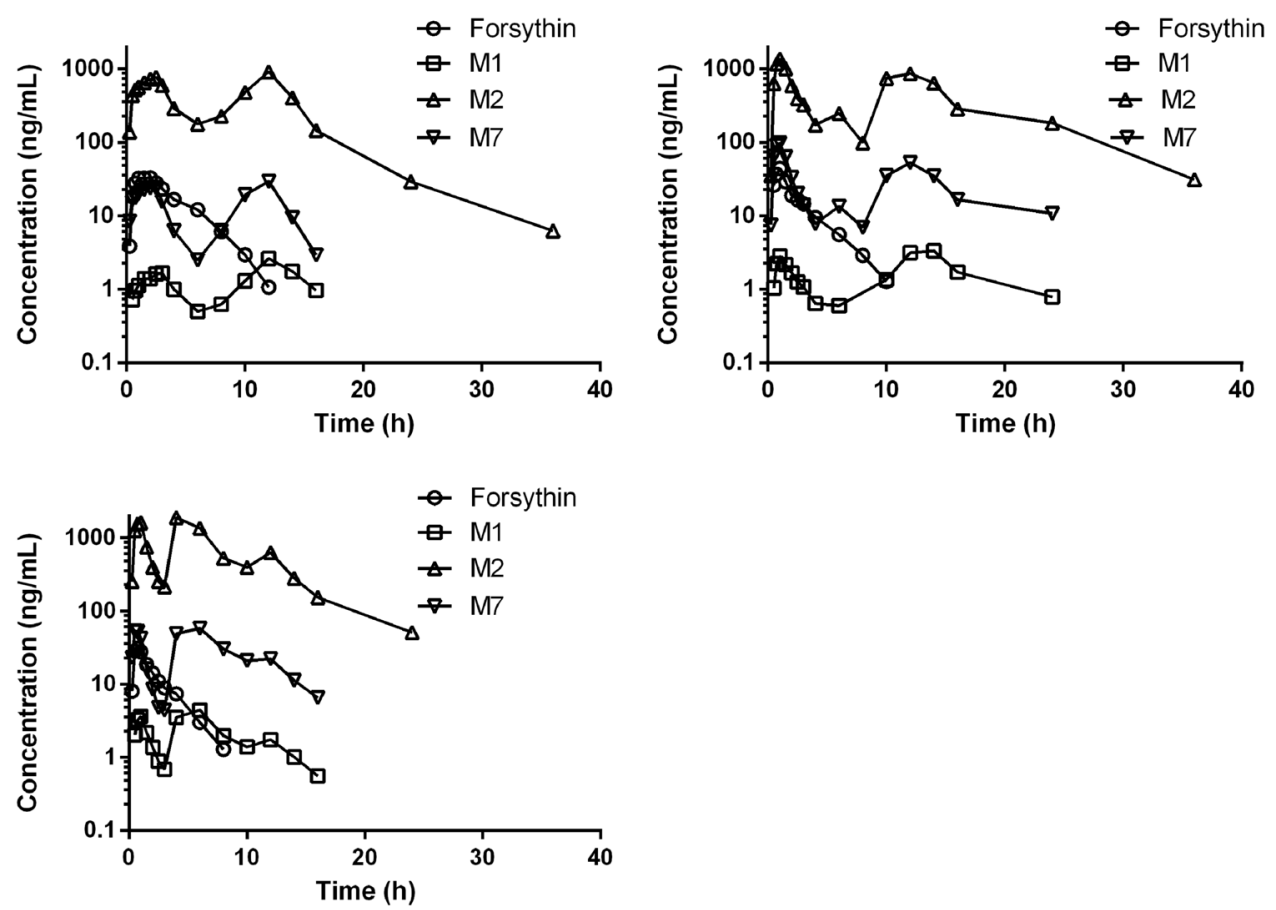

Fig. 4 Plasma concentration-time profiles of forsythin, M1 (aglycone), M2 (M1 sulfate), and M7 (M1 glucuronide) following administration of $100 \mathrm{mg}$ forsythin capsules in three healthy volunteers.
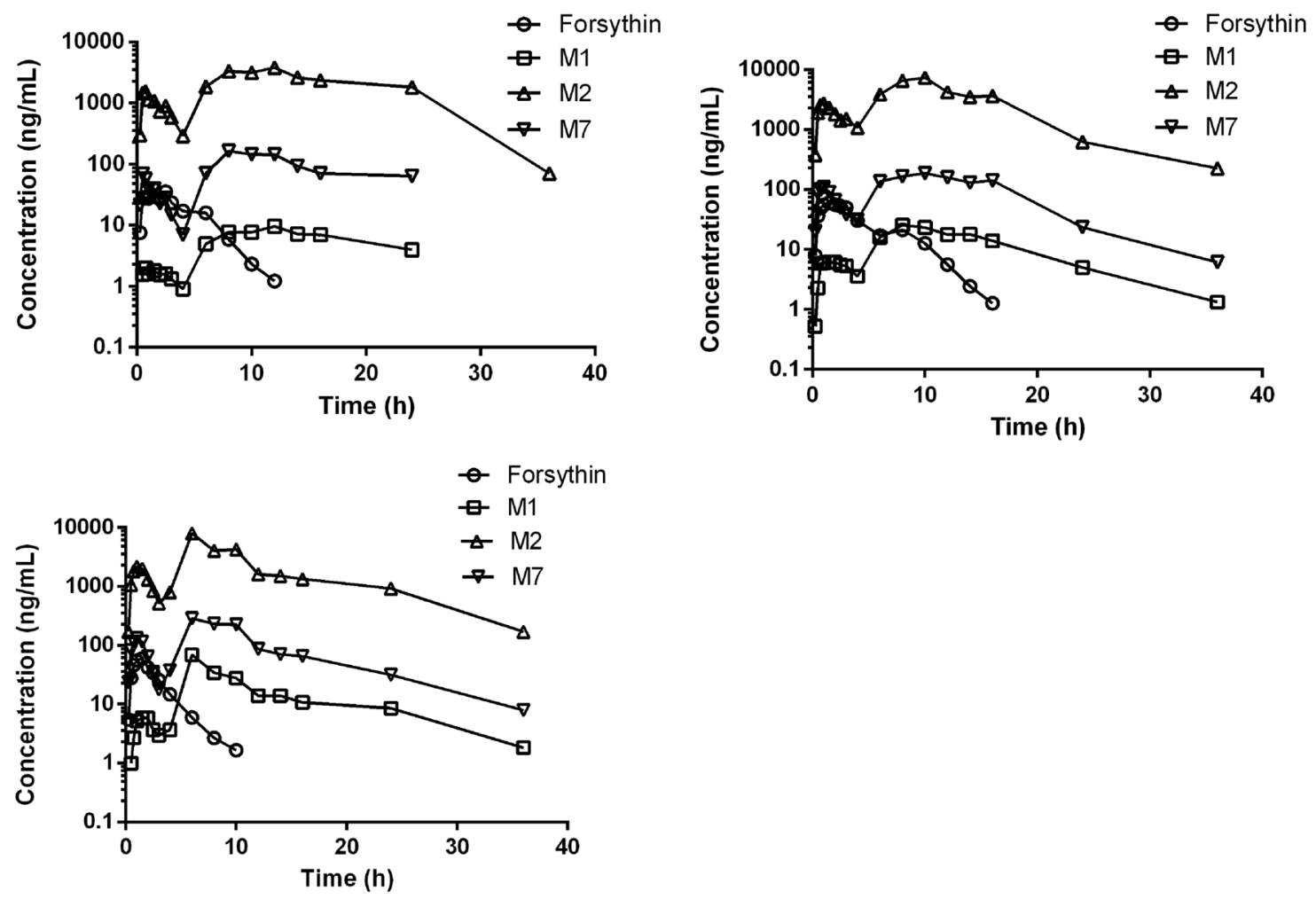

Fig. 5 Plasma concentration-time profiles of forsythin, M1 (aglycone), M2 (M1 sulfate), and M7 (M1 glucuronide) following administration of $800 \mathrm{mg}$ forsythin capsules in three healthy volunteers.

Urinary and fecal excretion of forsythin

A wide variety of metabolites, including sulfate conjugate, were detected in both urine and fecal samples, and the total urinary excretion accounted for $75.1 \%$ of the administered dose. The cumulative urinary excretion percentages of forsythin, M1, M2, and M7 in 8 healthy volunteers administered $100 \mathrm{mg}$ of forsythin are shown in Fig. 6. The cumulative excretion percentages of forsythin, M1, M2, and $M 7$ in urine samples were $0.752 \%$, $0.00416 \%, 71.6 \%$, and $2.79 \%$, respectively, while those of forsythin, $M 1, M 2$, and $M 7$ in fecal samples were $0,0.161 \%, 0.651 \%$, and 
Table 2. Pharmacokinetic parameters of forsythin, $M 1$ (aglycone), M2 (M1 sulfate), and M7 (M1 glucuronide) following administration of $100 \mathrm{mg}$ forsythin capsules in 8 healthy subjects.

\begin{tabular}{|c|c|c|c|c|}
\hline Parameters & Forsythin & M1 & M2 & M7 \\
\hline$C_{\max }(\mathrm{ng} / \mathrm{mL})$ & $31.8 \pm 6.5$ & $3.81 \pm 1.24$ & $1450 \pm 792$ & $72.2 \pm 55.1$ \\
\hline$T_{\max }(\mathrm{h})$ & $1.25(0.75-3.00)$ & $11.0(4.00-14.0)$ & $5.00(1.00-12.0)$ & $6.00(1.00-12.0)$ \\
\hline$C_{\max 1}(\mathrm{ng} / \mathrm{mL})$ & $29.7 \pm 8.23$ & $1.88 \pm 0.97$ & $893 \pm 462$ & $35.7 \pm 29.6$ \\
\hline$T_{\max 1}(\mathrm{~h})$ & $1.25(0.72-2.00)$ & $0.75(0.50-3.00)$ & $0.875(0.25-2)$ & $1.25(0.72-2.50)$ \\
\hline$C_{\max 2}(\mathrm{ng} / \mathrm{mL})$ & $22.0 \pm 13.6$ & $3.30 \pm 1.79$ & $1203 \pm 946$ & $59.8 \pm 58.3$ \\
\hline$T_{\max 2}(\mathrm{~h})$ & $2.50(3.00-6.00)$ & $6.00(2.50-12.0)$ & $6.00(3.00-12.0)$ & $6.00(2.50-12.0)$ \\
\hline$A \cup C_{0-t}(\mathrm{~h} \cdot \mathrm{ng} / \mathrm{mL})$ & $111 \pm 34$ & $31.3 \pm 11.8$ & $9700 \pm 1890$ & $417 \pm 132$ \\
\hline $\mathrm{AUC}_{0-\infty}(\mathrm{h} \cdot \mathrm{ng} / \mathrm{mL})$ & $114 \pm 34$ & $45.3 \pm 7.39$ & $9860 \pm 1960$ & $477 \pm 127$ \\
\hline$t_{1 / 2}(\mathrm{~h})$ & $1.75 \pm 0.26$ & $4.57 \pm 2.88$ & $4.36 \pm 1.61$ & $3.32 \pm 1.47$ \\
\hline $\mathrm{V}_{\mathrm{z}} / \mathrm{F}(\mathrm{L})$ & $2370 \pm 731$ & - & - & - \\
\hline $\mathrm{CL} / \mathrm{F}(\mathrm{L} / \mathrm{h})$ & $942 \pm 265$ & - & - & - \\
\hline
\end{tabular}

$C_{\max }$ maximum observed plasma concentration, $T_{\max }$ time of the maximum concentration, $A U C_{0-t}$ area under the concentration-time curve from time zero to the last measurable concentration, $A \cup C_{0-\infty}$ area under the concentration-time curve from $0 \mathrm{~h}$ to the infinite time, $t_{\max }$ time to the $C_{\max }, t_{1 / 2}$ apparent elimination half-life, $V_{z} / F$ apparent volume of distribution, $C L / F$ apparent clearance.

Table 3. Pharmacokinetic parameters of forsythin, M1 (aglycone), M2 (M1 sulfate), and M7 (M1 glucuronide) following administration of $800 \mathrm{mg}$ forsythin capsules in 8 healthy subjects.

\begin{tabular}{|c|c|c|c|c|}
\hline Parameters & Forsythin & M1 & M2 & M7 \\
\hline$T_{\max }(\mathrm{h})$ & $1.50(1.00-2.50)$ & $11.0(6.00-12.0)$ & $11.0(1.50-12.0)$ & $10.0(1.50-12.0)$ \\
\hline$T_{\max 1}(\mathrm{~h})$ & $1.25(0.50-2.50)$ & $0.875(0.50-1.50)$ & $0.875(0.50-1.50)$ & $0.75(0.5-1.5)$ \\
\hline$C_{\max 2}(\mathrm{ng} / \mathrm{mL})$ & $56.3 \pm 22.7$ & $19.8 \pm 20.8$ & $4275 \pm 2965$ & $235 \pm 95.8$ \\
\hline $\mathrm{AUC}_{0-\infty}(\mathrm{h} \cdot \mathrm{ng} / \mathrm{mL})$ & $283 \pm 80$ & $244 \pm 134$ & $59,400 \pm 19700$ & $3050 \pm 1060$ \\
\hline$t_{1 / 2}(\mathrm{~h})$ & $1.83 \pm 0.24$ & $7.00 \pm 2.29$ & $5.74 \pm 2.31$ & $6.6 \pm 3.55$ \\
\hline $\mathrm{V}_{\mathrm{z}} / \mathrm{F}(\mathrm{L})$ & $7920 \pm 2110$ & - & - & - \\
\hline $\mathrm{CL} / \mathrm{F}(\mathrm{L} / \mathrm{h})$ & $3070 \pm 1020$ & - & - & - \\
\hline
\end{tabular}

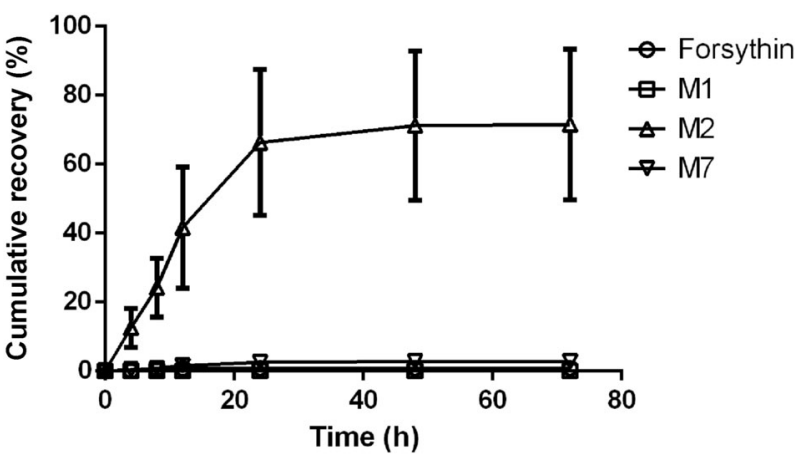

Fig. 6 Percentage of cumulative excretion of forsythin, M1 (aglycone), M2 (M1 sulfate), and M7 (M1 glucuronide) in urine after oral administration of $100 \mathrm{mg}$ of forsythin capsules in 8 healthy subjects.

$0.00502 \%$, respectively. Therefore, the sulfated conjugate $\mathrm{M} 2$ was excreted mainly through the kidneys, accounting for $71.6 \%$ of the administered dose, while the other related substances accounted for $\sim 3 \%$.
Identification of the SULT isoenzyme responsible for the sulfation of $\mathrm{M} 1$

To characterize the SULT isoforms associated with $M 2$ formation, the activities of SULT1A1*1, 1A1*2, 1A2,1A3,1B1, 1E1, and 2A1 were assessed. It was found that all 7 SULT isoforms could affect the yield of M2 (Fig. 7a). The formation rates of $M 2$ ranged from 244 to $4351 \mathrm{pmol} \cdot \mathrm{min}^{-1} \cdot \mathrm{mg}^{-1}$ protein, in descending order as follows: SULT1E1 $>1 A 1^{*} 1 \approx 1 A 2 \approx 1 A 1^{*} 2>1 B 1>1 A 3>2 A 1$ (Fig. $7 a$ ). After normalizing the rates of $M 2$ formation to the native contents of the SULT isoforms [9], sulfation of $M 2$ was found to be primarily catalyzed by SULT1A1 (Fig. 7b), followed by 1B1, 1E1, and 2A1. The SULT inhibitors DCNP $(1 \mu \mathrm{M})$, DCNP $(10 \mu \mathrm{M})$, and quercetin $(25 \mu \mathrm{M})$ suppressed M2 formation in HLCs by $72.1 \%, 87.6 \%$, and $94.9 \%$, respectively (Supplementary Fig. S6).

Identification of the UGT isoenzyme involved in glucuronidation of M1

To determine the main UGT isoenzymes involved in the glucuronidation of M1 to M7, human UGT phenotyping experiments were carried out. We investigated the formation of $M 7$ by 12 recombinant human UGT isozymes, namely, UGT1A1, 1A3, 1A4, $1 A 6,1 A 7,1 A 8,1 A 9,1 A 10,2 B 4,2 B 7,2 B 15$, and 2B17. As shown in Fig. 8, all 12 UGT recombinant enzymes could catalyze the 

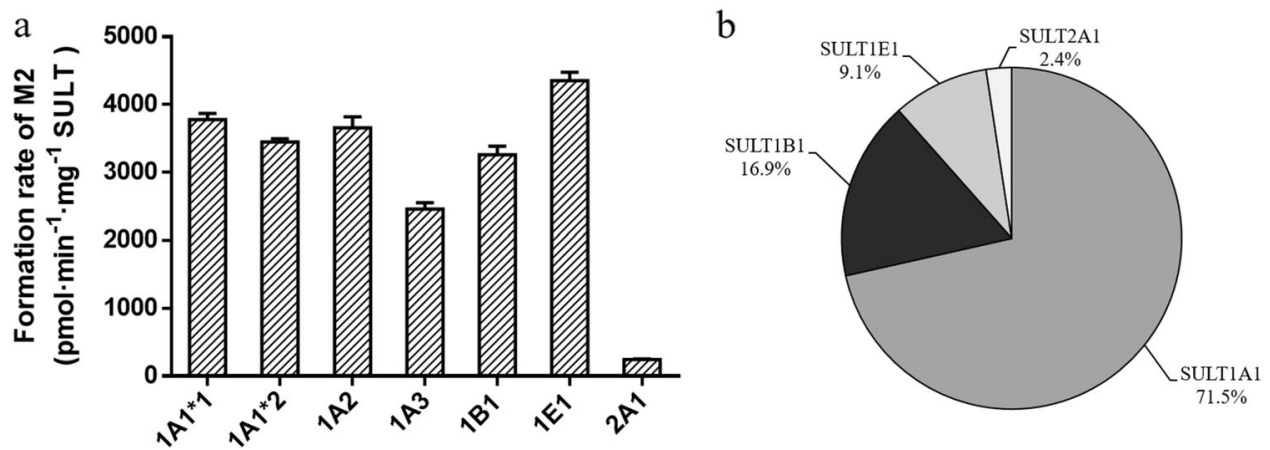

Fig. 7 Identification of the SULT isoenzyme responsible for the sulfation of M1. a Formation rate of M2 (M1 sulfate) in the incubation of M1 $(50 \mu \mathrm{M})$ with 7 human recombinant SULTs $(0.25 \mathrm{mg}$ protein $/ \mathrm{mL})$ supplemented with PAPS $(250 \mu \mathrm{M})$. b Contribution of each SULT isoform to the sulfation of M1 after normalization with regard to their native abundance in human liver cytosolic fraction.

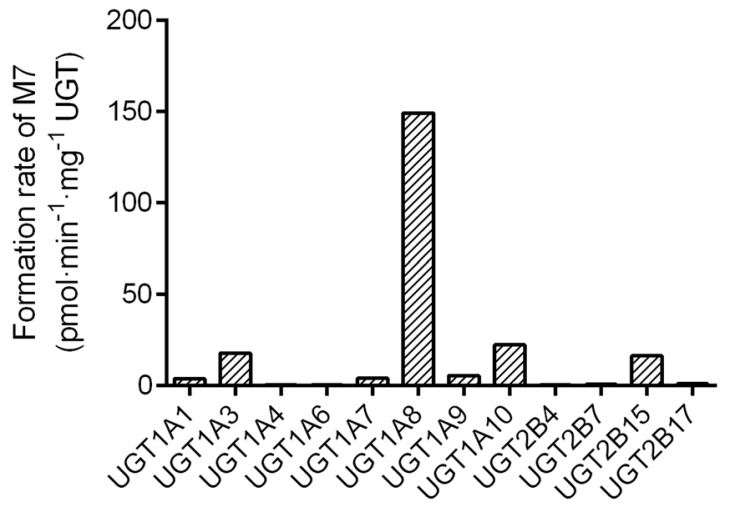

Fig. 8 Formation rate of $M 7$ ( $M 1$ glucuronide) in the incubation of M1 $(50 \mu \mathrm{M})$ with 12 human recombinant UGTs $(0.5 \mathrm{mg}$ protein $/ \mathrm{mL})$ supplemented with UDPGA $(2 \mathrm{mM})$.

transformation of M1 to M7. In the incubation systems of UGT1A4, $1 A 6,2 B 4,2 B 7$, and 2B17, the formation rates of $M 1$ glucuronide conjugates were all $<1.0 \mathrm{pmol} \cdot \mathrm{min}^{-1} \cdot \mathrm{mg}^{-1}$ protein. In the other UGT isoenzyme incubation systems, the formation rates ranged from 3.71 to $149 \mathrm{pmol} \cdot \mathrm{min}^{-1} \cdot \mathrm{mg}^{-1}$ protein, in descending order as follows: $\quad U G T 1 A 8>1 A 10>1 A 3 \approx 2 B 15>1 A 9 \approx 1 A 7 \approx 1 A 1$. As a result, the principal enzyme that contributed to the formation of glucuronide conjugate M7 was identified to be UGT1A8. Inhibition of the glucuronidation of $\mathrm{M} 1$ in HLMs by flurbiprofen was performed. The UGT inhibitor flurbiprofen (1 mM) suppressed M7 formation in HLMs by $75.4 \%$ (Supplementary Fig. S7).

Uptake of sulfated conjugate M2 by transfected HEK293 cells To evaluate the functions of OAT1, OAT3, and OCT2, an initial study was performed to compare the differences in positive substrates (PAH for OAT1, E3S for OAT3, and metformin for OCT2) in OAT1-, OAT3-, OCT2-, or mock-transfected HEK293 cells. Notably, the uptake rates of positive substrates in transportertransfected HEK293 cells were all greater than those in mocktransfected HEK293 cells (Fig. 9). Moreover, OAT1-, OAT3-, and OCT2-mediated uptake of PAH, E3S, and metformin, respectively, was remarkably inhibited by $200 \mu \mathrm{M}$ probenecid (OAT inhibitor) or $200 \mu \mathrm{M}$ TPA (OCT2 inhibitor).

To elucidate the roles of renal uptake transporters during sulfate conjugate M2 elimination, the activities of the three transporters (i.e., OAT1, OAT3, and OCT2) were determined. As presented in Fig. 9 , the uptake rate of $\mathrm{M} 2$ by OAT1 or OCT2 was relatively close to that of the mock cells (the ratio was $<2$ ), suggesting that $M 2$ is not a substrate of OAT1 or OCT2. The uptake rate of M2 by OAT3 was 22.1 times that of the mock-transfected cells, and this difference was statistically significant. In addition, probenecid decreased the uptake rate of M2 by OAT3 by $90 \%$, implying that M2 is an active substrate for OAT3. Nevertheless, the uptake rates of $\mathrm{M} 2$ were not markedly altered by other inhibitors, further showing that OAT1 and OCT2 are not responsible for M2 uptake.

Kinetic analysis of sulfation and glucuronidation The kinetic analysis of sulfation and glucuronidation of $\mathrm{M} 1$ both met with the Michaelis-Menten kinetics (Supplementary Fig. S8). The $K_{m}$ and $V_{\max }$ values are shown in Table 4 . Sulfation of $\mathrm{M} 1$ by SULT1A $1 * 1$ showed a markedly higher $V_{\max }$ value with a lower $K_{\mathrm{m}}$ value than M1 glucuronidation. The $V_{\max } / K_{\mathrm{m}}$ value of sulfation of $M 1$ was 1400 -fold higher than that of $M 1$ glucuronidation. The in vitro kinetic study provided direct evidence that M1 showed a preference for sulfation in vitro.

\section{DISCUSSION}

In this study, we evaluated the pharmacokinetics, metabolism, and excretion routes of forsythin. We also evaluated the roles of metabolizing enzymes involved in the metabolism of aglycone M1. Our results demonstrated that forsythin could undergo extensive metabolism in humans and generated a total of 13 metabolites in plasma, urine and feces. However, compared with $M 2$, the contents of the other metabolites were all lower than $10 \%$, further suggesting that M2 was the principal metabolite of forsythin. Based on available pharmacodynamic data, both M1 and $M 2$ were found to be active. The activity of $M 2$ is higher than that of M1 but lower than that of forsythin. It is worth noting that enterohepatic circulation can occur through intestinal reabsorption and biliary excretion, often accompanied by intestinal deconjugation and hepatic conjugation. In general, it may prolong the pharmacologic actions of specific drugs and drug metabolites [10]. In this study, as shown in Figs. 4 and 5, enterohepatic recycling was observed in the plasma concentration-time profiles of $M 1, M 2$, and $M 7$. At the other doses, enterohepatic recycling was also demonstrated (Supplementary Figs. S2-S5). We inferred that after M1 was reabsorbed by the small intestine, it was quickly sulfated to form $\mathrm{M} 2$ since the small intestine contains a large amount of SULTs, with SULT1B1 (36\%) and SULT1A3 (31\%) being the major enzymes present [9]. The liver is not the only organ containing UGTs, and the distribution of UGTs in each tissue is different. Thus, a second peak on the metabolite curves was observed. In addition, the half-life values of $M 2$ and $M 7$ were 4-6 h, which were longer than those of the parent drug.

Glucuronidation is a pivotal metabolic pathway of some drugs and endogenous substances in humans. Glucuronidation of phenols usually occurs with nucleophilic groups such as hydroxyl groups [11]. In addition, numerous drugs (e.g., morphine) and 


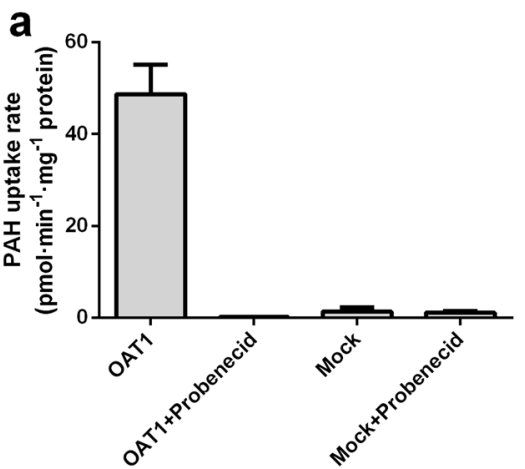

b
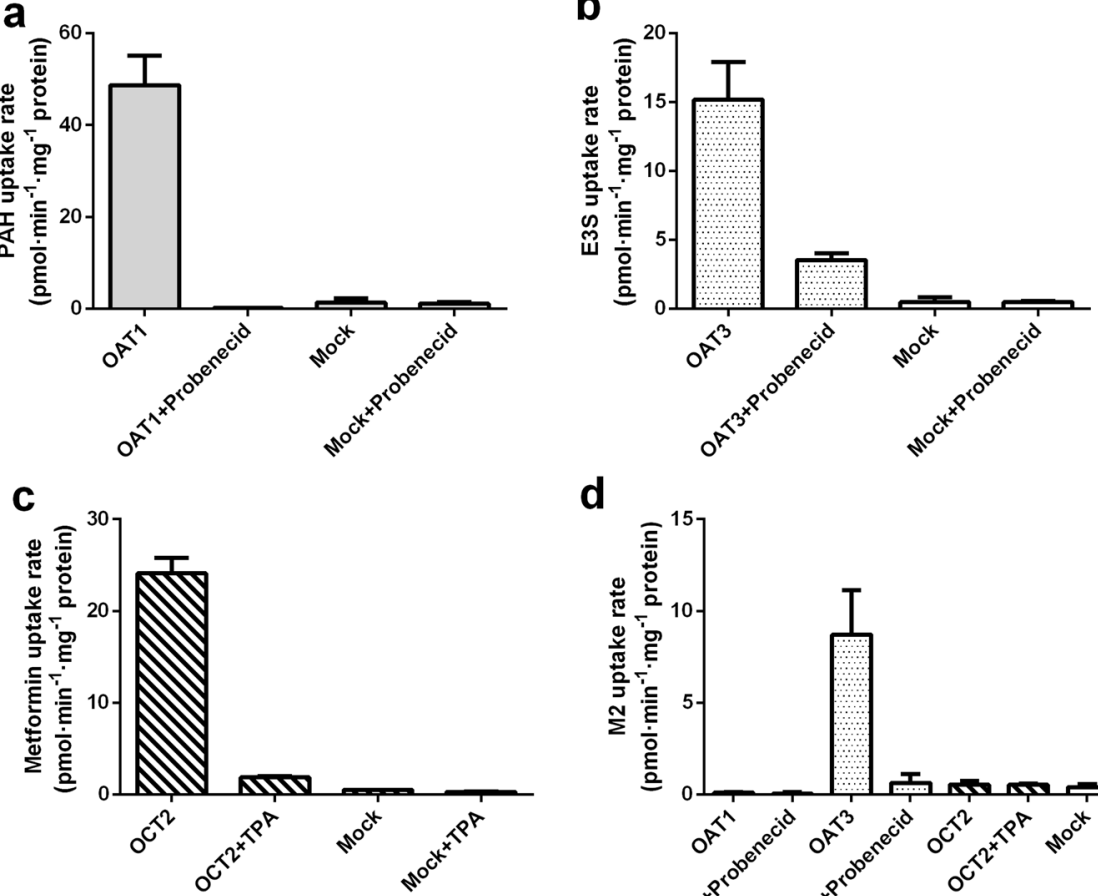

d

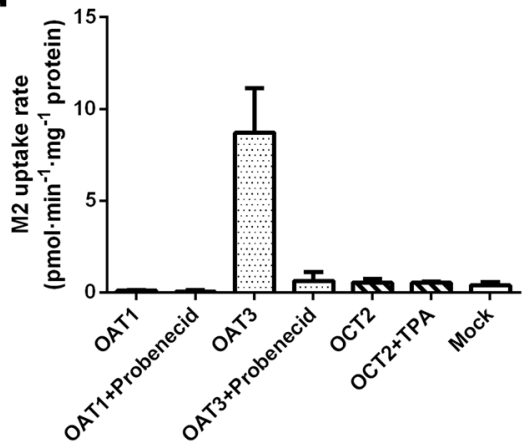

Fig. 9 Uptake of standard substrates and test compound in the absence or presence of specific transporter inhibitors in transportertransfected and mock HEK293 cells. Substrates used. a PAH; b E3S; c metformin; d aglycone sulfate conjugate M2 (M1 sulfate).

Table 4. Kinetic parameters of $M 1$ sulaftion in human recombinant SULT1A $1 * 1$ and M1 glucuronidation in human recombinant UGT1A8.

\begin{tabular}{lll}
\hline Incubation system & SULT1A $1{ }^{* 1}$ & UGT1A8 \\
\hline Protein concentration $(\mathrm{mg}$ protein $/ \mathrm{mL})$ & 0.0250 & 0.100 \\
Incubation time $(\mathrm{min})$ & 15.0 & 60.0 \\
Concentration range $(\mu \mathrm{M})$ & $0.200-20.0$ & $2.00-250$ \\
$K_{\mathrm{m}}(\mu \mathrm{M})$ & 3.68 & 63.6 \\
$V_{\max }\left(\mathrm{pmol} \cdot \mathrm{min}^{-1} \cdot \mathrm{mg}^{-1}\right.$ protein) & 44,391 & 548 \\
$V_{\text {max }} / K_{\mathrm{m}}\left(\mu \mathrm{L} \cdot \mathrm{min}^{-1} \cdot \mathrm{mg}^{-1}\right.$ protein) & 12,062 & 8.61 \\
\hline
\end{tabular}

flavonoid glycosides (e.g., puerarin) directly undergo glucuronidation [12-14].

Similar to glucuronidation, sulfation is also a common phase II reaction that occurs during the activation and detoxification of various exogenous and endogenous compounds in humans $[15,16]$. Typically, sulfation displays a high affinity and low capacity for xenobiotic conjugation, while glucuronidation acts in the opposite way. Acetaminophen is a xenobiotic substrate for both SULTs and UGTs. The sulfation and glucuronidation abilities of acetaminophen are dose-dependent. Acetaminophen sulfate is the principal conjugate metabolized by SULTs at low doses. With an increasing dose of acetaminophen, the percent conjugated with sulfate decreased, while that conjugated with glucuronic acid increased. However, considering the substrate inhibition of SULT, the absolute amount of the sulfate conjugate can decrease at high doses. This phenomenon has also been observed in the Caco-2 model. The excretion of apigenin glucuronide at high concentrations $(>25 \mu \mathrm{M})$ has been reported, while that of the sulfate conjugate is more significant at low concentrations $[17,18]$. However, in our study, the sulfate conjugate $M 2$ constituted the principal circulating metabolite rather than glucuronide conjugate $M 7$, and $M 2$ dominated the pharmacokinetics, metabolism, and excretion of forsythin within the 50-800 mg dosing range.

According to the pharmacokinetic results, M2 was more easily generated than M7. To verify this conjecture and characterize the phenotypes of the major metabolizing enzymes, further experiments were carried out. In vitro phenotyping experiments revealed that SULT1A1*1, 1A1*2, 1A2, 1A3, 1B1, 1E1, and 2A1 could all mediate the transformation of $M 1$ to $M 2$, of which SULT1A1 represented the main subtype for $M 1$ sulfation. In addition, we assessed the roles of UGT during M7 formation at the same substrate concentration $(50 \mu \mathrm{M})$ because the exposure of M7 was greater than that of the parent drug. Our findings indicated that the glucuronidation of M1 was catalyzed mainly by UGT1A8, and its formation rate was $149 \mathrm{pmol} \cdot \mathrm{min}^{-1} \cdot \mathrm{mg}^{-1}$ protein. Although all 12 UGT subtypes could mediate the transformation of $\mathrm{M} 1$ to $\mathrm{M} 7$, the formation rate of $\mathrm{M} 1$ to $\mathrm{M} 7$ mediated by UGT was much lower than that of M1 to M2 mediated by SULT. This indicated that M1 preferred sulfation to glucuronidation, which also explained to some extent the large amounts of $M 2$ that were detected instead of M7. In addition, the in vitro kinetic study showed that M1 had greater affinity for SULT1A1 than UGT1A8, providing direct evidence that $M 1$ more easily generated $M 2$ than M7 in vitro. One volunteer was orally administered $100 \mathrm{mg}$ of aglycon M1. The main drug-related component in his plasma was sulfate-conjugated $M 2$ instead of $M 7$, further confirming that whether the concentration of aglycon $\mathrm{M} 1$ was low or high, sulfation played a dominant role in the phase II reactions of M1. Thus, after oral administration or injection of Shuanghuanglian, the main component in the human body will be totally different. After oral administration of Shuanghuanglian, forsythin undergoes first-pass metabolism mainly via SULT1A1, leading to $M 2$ as the main drug-related component in systemic circulation. Our previous work has shown that the human plasma protein binding rates of forsythin, $\mathrm{M} 2$, and $\mathrm{M} 7$ are $69.2 \%, 79.4 \%$, and $94.7 \%$, respectively. 
Human SULT isoenzyme phenotyping experiments revealed that SULT1A1 significantly contributed to the formation of M2. There are three common genetic variants in the SULT1A1 gene: SULT1A $1 * 1,1 A 1 * 2$, and $1 A 1 * 3[19,20]$. Although the activity of SULT1A $1 * 2$ is $40 \%$ lower than that of SULT1A $1 * 1$ for the sulfation of troglitazone [21], relatively similar activity levels of SULT1A1*1 and $1 A 1 * 2$ were noted during $M 2$ formation (Fig. 7a). Nonetheless, the activity of SULT1A $1 * 3$ was not examined due to its commercial unavailability.

Considering that the sulfate conjugate $\mathrm{M} 2$ is a hydrophilic compound with a clogD value of -2.41 , which was calculated using ACD software version 14.0 (ACD/Labs, Advanced Chemistry Development, Inc., Toronto, ON, Canada), it does not readily pass through biological membranes via passive diffusion. In addition, $\sim 75.1 \%$ of the dose was recovered in urine, with $\mathrm{M} 2$ accounting for $71.6 \%$ of the dose. Therefore, we further examined the uptake of $\mathrm{M} 2$ by the transporters OAT1, OAT3, and OCT2. Members of the OAT family, particularly OAT1 and OAT3, are responsible for the renal transport of organic anions. Members of the OCT family are involved in the transport of various xenobiotics as well as endogenous organic cations [8]. In this study, the uptake rate of M2 by OAT1 and OCT2 was less than 2-fold, suggesting that M2 may not be transported by OAT1 and OCT2. Notably, the uptake rate of M2 was markedly higher in HEK293 cells transfected with OAT3 than in mock-transfected cells. The findings of OAT inhibition with probenecid further verified that OAT3 was the principal transporter of M7. We also carried out uptake experiments of M7 by transfected HEK293 cells. The results showed that M7 is also a substrate of OAT3. Secretory transporters are also expressed on the apical membrane, such as multidrug and toxin extrusion transporter 1 (MATE1), breast cancer resistance proteins $(\mathrm{BCRP})$, and multidrug resistance associated proteins 2 and 4 (MRP2 and MRP4) [22]. Further studies should therefore be conducted.

Clinically, many drugs are substrates for OAT1 and/or OAT3. In patients with chronic renal insufficiency, these drugs can exhibit certain pharmacokinetic changes, such as decreased renal clearance and increased plasma exposure, due to the obstruction of renal excretion. In a chronic renal insufficiency state, the expression or function of transporters may be altered [23]. For instance, the sulfated conjugate of morinidazole is a sensitive substrate of OAT1 and OAT3, whereas the glucuronides are limited to OAT3 only. In patients with chronic kidney failure, the three conjugates resulted in a 15-fold increase in total plasma exposure to the drug. Moreover, the conjugates may exert pharmacologic or adverse effects [24]. For tenofovir, its plasma exposure increased by 8.5 -fold in patients with end-stage renal disease [25].

In conclusion, our study reveals that forsythin is well absorbed and extensively metabolized following oral administration, and the sulfate conjugate $\mathrm{M} 2$ is the main metabolite in plasma. Moreover, renal excretion is the principal excretion pathway for $M 2$, and $M 2$ is a substrate for OAT3. In addition, SULT1A1 predominantly mediates the sulfation of M1, while UGT1A8 is the primary enzyme involved in glucuronidation. The rate of M1 sulfation was much higher than that of glucuronidation. The significantly greater exposure and plasma concentration of M2 compared with the parent drug indicate that severe renal impairment patients may be subjected to a remarkable increase in the plasma concentration of $\mathrm{M} 2$ compared with healthy subjects. Therefore, the safety of forsythin in renally impaired patients should be placed under close monitoring.

\section{ACKNOWLEDGEMENTS}

This study was supported by the National Natural Science Foundation of China (grant nos. 81521005 and 81903701) and the National Key Research Project of the Chinese Academy of Sciences (grant no. XDA12050306).

\section{AUTHOR CONTRIBUTIONS}

LLP, XXD, LF, and DFZ participated in research design. LLP and YY conducted experiments. $\mathrm{MH}, \mathrm{SW}$, and LF contributed new reagents or analytic tools. LLP and YY performed data analysis. YHD, CYL, and $\mathrm{HZ}$ participated in clinical trials. LLP, XXD, and DFZ contributed to the writing of the paper.

\section{ADDITIONAL INFORMATION}

The online version of this article (https://doi.org/10.1038/s41401-020-0481-8) contains supplementary material, which is available to authorized users.

Competing interests: The authors declare no competing interests. The authors alone are responsible for the content and writing of this article.

\section{REFERENCES}

1. Wang Z, Xia Q, Liu X, Liu W, Huang W, Mei X, et al. Phytochemistry, pharmacology, quality control and future research of forsythia suspensa (Thunb.) Vahl: a review. J Ethnopharmacol. 2018;210:318-39.

2. Ozaki Y, Rui J, Tang Y, Satake M. Antiinflammatory effect of forsythia suspensa vahl and its active fraction. Biol Pharm Bull. 1997;20:861-64.

3. Chinese Pharmacopoeia Commission. Pharmacopoeia of the People's Republic of China. China Medical Science Press; 2015.

4. Han Z, Lei XL, Zhang H, Liu L, Chen ZS, Yang W, et al. Evaluating the safety of forsythin from forsythia suspensa leaves by acute and sub-chronic oral administration in rodent models. Asian Pac J Trop Med. 2017;10:47-51.

5. Rowland A, Miners JO, Mackenzie PI. The UDP-glucuronosyltransferases: their role in drug metabolism and detoxification. Int J Biochem Cell Biol. 2013;45:1121-32.

6. Suiko M, Kurogi K, Hashiguchi T, Sakakibara Y, Liu MC. Updated perspectives on the cytosolic sulfotransferases (SULTs) and SULT-mediated sulfation. Biosci Biotechnol Biochem. 2017;81:63-72.

7. Chinese Pharmacopoeia Commission. Guidance for bioanalytical method validation, Pharmacopoeia of the People's Republic of China. China Med Sci Press. 2015:4:363-8.

8. Zhong K, Li XL, Xie C, Zhang YF, Zhong DF, Chen XY. Effects of renal impairment on the pharmacokinetics of morinidazole: uptake transporter-mediated renal clearance of the conjugated metabolites. Antimicrob Agents Chemother. 2014;58:4153-61.

9. Riches Z, Stanley EL, Bloomer JC, Coughtrie MW. Quantitative evaluation of the expression and activity of five major sulfotransferases (SULTs) in human tissues: the SULT "pie". Drug Metab Dispos. 2009;37:2255-61.

10. Roberts MS, Magnusson BM, Burczynski FJ, Weiss M. Enterohpatic circulation. Clin Pharmacokinet. 2002;41:751-90.

11. Wu B, Kulkarni K, Basu S, Zhang S, Hu M. First-pass metabolism via UDP-glucuronosyltransferase: a barrier to oral bioavailability of phenolics. J Pharm Sci. 2011;100:3655-81.

12. Yang G, Ge S, Singh R, Basu S, Shatzer K, Zen M, et al. Glucuronidation: driving factors and their impact on glucuronide disposition. Drug Metab Rev. 2017;49:105-38.

13. Andersen G, Christrup L, Sjøgren P. Among morphine metabolism, pain and side effects during long-term treatment: an update. J Pain Symptom Manag. 2003;25:74-91.

14. Luo CF, Cai B, Hou N, Yuan M, Liu SM, Ji H, et al. UDP-glucuronosyltransferase 1A1 is the principal enzyme responsible for puerarin metabolism in human liver microsomes. Arch Toxicol. 2012;86:1681-90.

15. Tian $X$, Huo $X$, Dong $P$, Wu $B$, Wang $X$, Wang $C$, et al. Sulfation of melatonin: enzymatic characterization, differences of organs, species and genders, and bioactivity variation. Biochem Pharmacol. 2015;94:282-96.

16. Barbosa ACS, Feng Y, Yu C, Huang M, Xie W. Estrogen sulfotransferase in the metabolism of estrogenic drugs and in the pathogenesis of diseases. Expert Opin Drug Metab Toxicol. 2019;15:329-39.

17. Liu Y, Hu M. Absorption and metabolism of flavonoids in the Caco-2 cell culture model and a perused rat intestinal model. Drug Metab Dispos. 2002;30:370.

18. Hu M, Chen J, Lin H. Metabolism of flavonoids via enteric recycling: mechanistic studies of disposition of apigenin in the Caco-2 cell culture model. J Pharmacol Exp Ther. 2003;307:314-21.

19. J Carlini E, B Raftogianis R, Wood T, Jin F, Zheng W, Rebbeck T, et al. Sulfation pharmacogenetics: SULT1A1 and SULT1A2 allele frequencies in Caucasian, Chinese and African-American subjects. Pharmacogenetics. 2001;11:57-68.

20. Rasool MI, Bairam AF, Gohal SA, El Daibani AA, Alherz FA, Abunnaja MS, et al Effects of the human SULT1A1 polymorphisms on the sulfation of acetaminophen, O-desmethylnaproxen, and tapentadol. Pharmacol Rep. 2019;71: 257-65. 
21. Honma W, Shimada M, Sasano H, Ozawa S, Miyata M, Nagata K, et al. Phenol sulfotransferase, ST1A3, as the main enzyme catalyzing sulfation of troglitazone in human liver. Drug Metab Dispos. 2002;30:944-49.

22. Morrissey KM, Stocker SL, Wittwer MB, Xu L, Giacomini KM. Renal transporters in drug development. Annu Rev Pharmacol Toxicol. 2013;53:503-29.

23. Schwenk MH, Pai AB. Drug transporter function-implications in CKD. Adv Chronic Kidney Dis. 2016;23:76-81.
24. Kong FD, Pang XY, Zhong K, Guo ZT, Li XL, Zhong DF, et al. Increased plasma exposures of conjugated metabolites of morinidazole in renal failure patients: a critical role of uremic toxins. Drug Metab Dispos. 2017;45: 593-603.

25. Kearney BP, Yale K, Shah J, Zhong L, Flaherty JF. Pharmacokinetics and dosing recommendations of tenofovir disoproxil fumarate in hepatic or renal impairment. Clin Pharmacokinet. 2006;45:1115-24. 